\title{
Incoherent Bright Field STEM for Imaging and Tomography of Ultra-Thick TEM Cross-sections
}

\author{
Peter Ercius* and David Muller*
}

*School of Applied and Engineering Physics, Cornell University, E13 Clark Hall, Ithaca, NY, 14853

Typical high resolution images acquired by a scanning transmission electron microscope (STEM) require specimen thicknesses $<40 \mathrm{~nm}$ to avoid significant image blurring due to beam spreading, but images of much thicker cross-sections can provide analysis of extended structures in their entirety at the expense of resolution. Such projected images, sometimes up to several microns in thickness, are useful inputs for electron tomography provided the signal is monotonic with projected thickness and a sufficient signal-to-noise ratio (SNR) is maintained. Annular dark field (ADF) STEM imaging is a very effective method for samples thinner than $50-100 \mathrm{~nm}$, but can produce contrast reversal artifacts in thicker samples [1]. Incoherent bright field (IBF) STEM imaging can correct these problems, but requires a greater dynamic range on the detector [1]. We use the SNRs of the ADF and IBF STEM techniques as a measure for image quality to produce a general relationship determining when each method is better suited for imaging any material at a given thickness.

An ADF-STEM detector collects only the highly scattered electrons to suppress diffraction contrast and to provide directly interpretable image intensities that vary with a material's atomic number $Z$. An ideal ADF detector would collect all highly scattered electrons, but physical limitations inside the microscope column allow electrons to scatter beyond the outer collection diameter of the detector (including backscatter). Loss of a majority of electrons due to high angle scattering produces a contrast reversal that deviates from the expected monotonic relationship and can produce image artifacts making the densest parts of the sample appear as voids. The contrast reversals are especially noticeable in low voltage STEM, even at moderate sample thicknesses.

One approach to obtaining a monotonic signal and avoid contrast reversals is the IBF STEM technique, which has proven useful for imaging ultra-thick cross-sections of crystalline materials [1]. An IBF detector collects all electrons scattered between $0-100 \mathrm{mrad}$ to suppress diffraction contrast similarly to ADF STEM but also produces monotonic image intensities at all material thicknesses. Figure 1 shows a line profile of IBF-STEM intensities that monotonically decreases for $200 \mathrm{keV}$ electrons transmitted through a Ta wedge continually increasing in thickness, while the ADF signal undergoes a contrast reversal.

The high energy incident electrons have estimated total path lengths $>>10 \mathrm{~m}$ in most materials [2], and it is therefore reasonable to assume that all incident electrons $\mathrm{I}_{0}$ exit from the specimen. All scattering from the material could then be designated as high-angle ADF (HAADF) scattering $(>100 \mathrm{mrad})$ for an ideal ADF detector or low-angle IBF scattering $(<100 \mathrm{mrad})$, and MonteCarlo simulations of electron scatter from various materials are used to compare the expected SNRs produced from these two scattering regimes. The full unscattered electron beam is always incident on the IBF detector and produces a large noise component that overwhelms intensities for thin material, but HAADF avoids the central beam providing lower noise in equivalent conditions. As material thickness increases, more electrons scatter out of the central beam oppositely affecting the noise of each technique, and the IBF- and ADF-STEM SNRs are equal at a material thickness $(t)$ 
where $50 \%$ of all electrons scatter to high angles. This thickness is directly related to the elastic mean free path $\left(\lambda_{e}\right)$ of electrons in the material by Beer's law. A simple estimation of $\lambda_{e}$ by the Rutherford scattering cross-section with empirically determined $Z^{1.7}$ dependence [3] is therefore plotted against $t$ as determined by Monte-Carlo simulations of $200 \mathrm{keV}$ incident electrons for various elements. The resulting linear relationship shown in Figure 2 provides as estimation of the cutoff thickness $t$ at which IBF-STEM yields superior SNR for any target material according to

$$
t \propto\left(\frac{A_{t}}{Z^{1.7} \rho}\right)
$$

where $A_{t}, Z$ and are the atomic weight, atomic number and density of the target material.

Previously, the IBF technique was utilized only as a replacement for ADF-STEM imaging to provide artifact free projection images of ultra-thick cross-sections possibly affected by contrast reversal. We show that IBF imaging conditions are superior in ultra-thick samples even before ADF-STEM reverses contrast, which provides well defined material feature boundaries for accurate structural measurements. [4]

[1] P. Ercius, et al., Applied Physics Letters, 88, (2006) 243116.

[2] L. Pages, et al, Atomic Data, 4, (1972) 1-27.

[3] E.J. Kirkland, et al., Ultramicroscopy, 23 (1987) 77.

[4] Funding and support provided by the Semiconductor Research Corporation and Cornell Center for Materials Research.

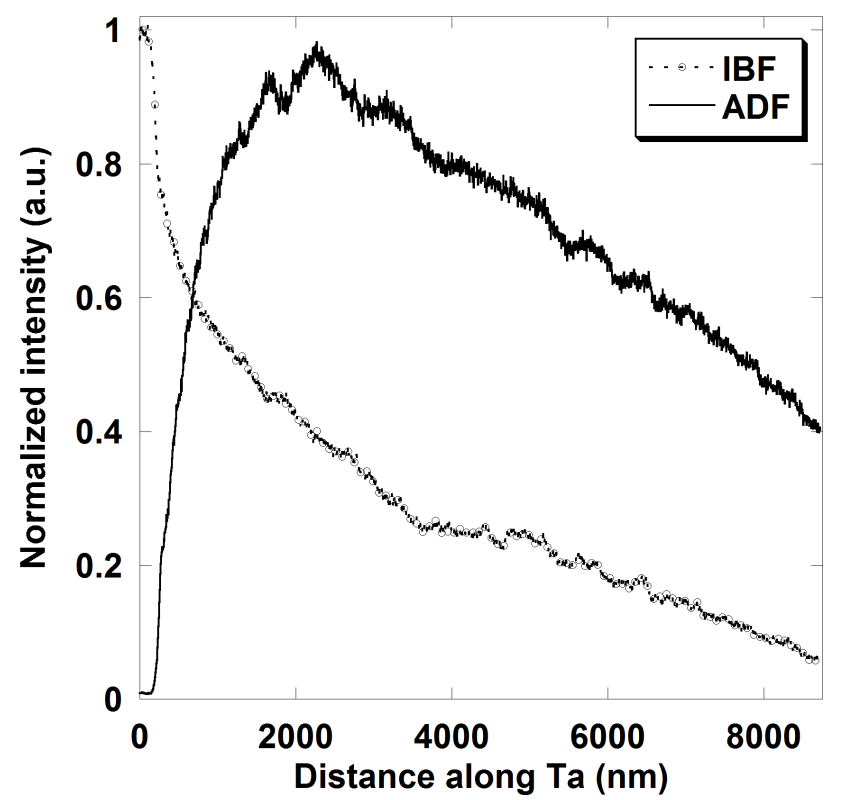

Figure 1. Line profiles along a Ta wedge with a continuously increasing projected thickness showing the experimental transmitted electron intensities for incoherent bright field (IBF) and annular dark field (ADF) STEM. The ADF-STEM reverses contrast, but the IBF-STEM provides monotonic transmitted electron intensities at all thicknesses.

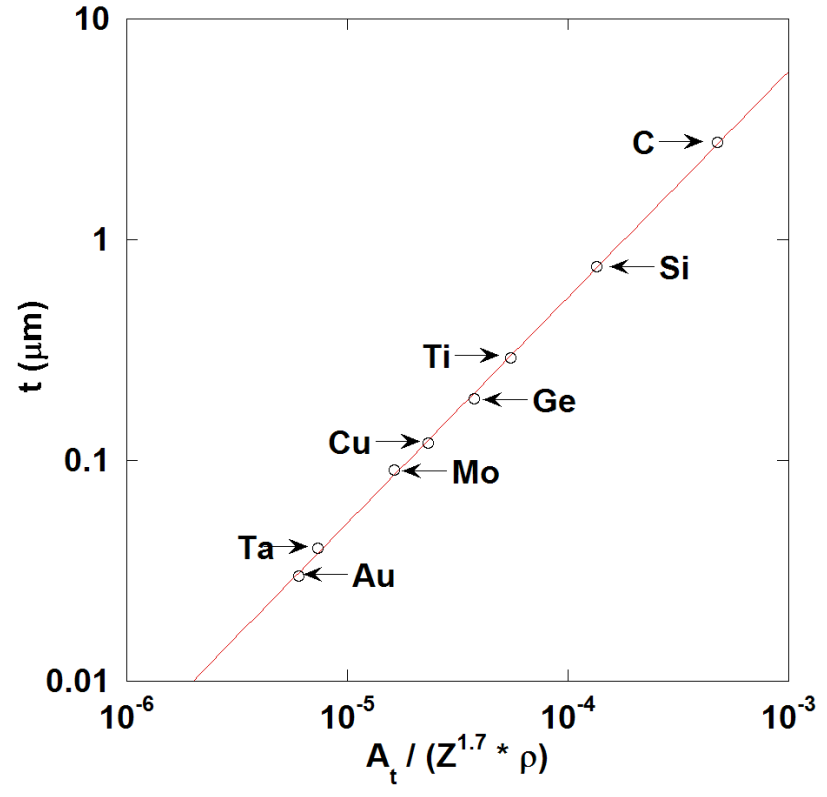

Figure 2. A log-log plot of the estimated material Rutherford cross-section with empirical $Z^{1.7}$ dependence against the thickness $t$ at which the ADF and IBF signal-tonoise ratios (SNR) are equal for various elements. The linear relationship (see equation 1) provides an estimation for the cutoff thickness for any material above which the IBF-STEM technique provides higher quality images compared to the ADF-STEM technique. 